$\Delta$ Palabras clave/ Arquitectura moderna biopolítica, Frente Popular, Luciano Kulczewski.

$\Delta$ Keywords/ Modern architecture, biopolitics, Popular Front, Luciano Kulczewski.

$\Delta$ Recepción/ 3 abril 2018

$\Delta$ Aceptación/ 7 junio 2018

\title{
Edificios máquinas: Arquitectura Moderna y disciplinaria del Frente Popular en Tocopilla ${ }^{1}$
}

\section{Machinery Buildings: Disciplinary and Modern Architecture of the Popular Front in Tocopilla ${ }^{1}$}

\section{Damir Galaz-Mandakovic}

Profesor de Historia, Universidad de Tarapacá, Chile.

Magíster en Antropología, Universidad Católica del Norte, Chile.

Magister en Ciencias Sociales, Universidad de Antofagasta, Chile.

Doctor en Historia, Université Rennes 2, Francia. Doctor en Antropología, Universidad Católica de Norte, Chile.

damirgalaz@gmail.com

RESUMEN/ En el presente artículo se caracteriza un proceso de producción arquitectónica sustentada en el Movimiento Moderno en la ciudad de Tocopilla, durante el gobierno del Frente Popular. Se examinan las dimensiones urbana, social, arquitectónica y biopolítica como horizontes ideológicos que activaron la reacción gubernamental frente a una crisis socioeconómica. ABSTRACT/ This article describes an architecture production process supported by the Modern Movement in the city of Tocopilla during the Popular Front's administration. Urban, social, architectural and biopolitical aspects are discussed as ideological horizons that triggered governmental action facing a socioeconomic crisis.

\section{INTRODUCCIÓN.} que se relacionan con la arquitectura y las gestiones de la masividad deben ser estudiados desde diversos enfoques. En ese sentido, el diálogo entre la historia de la arquitectura y la historia de los procesos económicos, sociales y políticos, no excluye incorporar una mirada antropológica respecto a etapas significativas de la historia del norte de Chile. Dichas etapas están relacionadas con cambios paradigmáticos que incluyeron nuevas escalas urbanas, adicionando originales miradas respecto a la subalternidad, significando, finalmente, una innovación relacional entre el Estado y el mundo popular, relación que, en nuestro caso de estudio, estuvo mediada por la arquitectura moderna en el marco de una crisis socioeconómica en la ciudad de Tocopilla (Región de Antofagasta, Chile). En ese contexto, desde un enfoque multidisciplinario, nos preguntamos: ¿cómo respondió la arquitectura a los cambios que se atestiguaron en el norte de Chile una vez que el ciclo del salitre Shanks colapsó? ¿Qué rol tuvo la arquitectura moderna en la gestión de una masividad migrante y desplazada por la pobreza en la zona salitrera cercana a Tocopilla? ¿Qué herramientas conceptuales nos pueden ayudar a comprender los procesos de implementación arquitectónica y planificaciones urbanas en cuanto a política y control social?
CRISIS Y DESPLAZADOS. El informe World Economic Survey (League of Nations 1933), indicó que Chile fue el país más afectado por la Gran Depresión de 1929 iniciada en EE.UU.: las exportaciones de salitre y cobre se derrumbaron provocando graves consecuencias sobre la economía interna. Al caer los ingresos fiscales y disminuir las reservas, se profundizaron los impactos que ya se venían arrastrando por la producción de salitre sintético en Alemania y por la obsolescencia técnica del Sistema Shanks.

El cierre de las salitreras no solo generó el fin de un ciclo técnico y productivo en la minería no metálica a contar de 1930, sino que también trajo consigo un denso éxodo de cesantes que bajaron a los puertos para buscar nuevos horizontes. Muchos querían volver a sus zonas de origen, generalmente la zona central del país. Por citar una 
muestra aleatoria, podemos ver que con fecha 10 de mayo de 1932 se embarcaron en los vapores Taltal y Fresia 394 cesantes del salitre, quienes, junto a sus familias, sumaron un total de 719 personas. El 13 de mayo de 1932 se informaba en un acta de la Gobernación Marítima de Tocopilla que los vapores Huasco y Margara llevaban 432 cesantes los que, sumados a sus familias, alcanzaban las 881 personas (La Prensa de Tocopilla 1932).

De esta forma, Tocopilla, además de constituirse como un pasillo, también se constituyó en la ciudad que tuvo que recibir a miles de cesantes que comenzaron una nueva vida en el puerto. El recibimiento de una gran cantidad de cesantes, generó un caos urbano por efecto de los problemas sanitarios y una ocupación del espacio descontrolado, surgiendo una infinidad de barrios improvisados. En ese escenario, afloraron enfermedades que diezmaron la población, entre ellas la fiebre tifoidea y el tifus exantemático. En septiembre de 1932, la Dirección de Sanidad de Tocopilla ordenó una severa higienización de la "viviendas insalubres", además del "despiojamiento" y "baño de los vagos, pordioseros, vendedores ambulantes, escolares y cesantes. La desratización intensiva con venenos y trampas" (Archivo de la Gobernación de Tocopilla, oficio № 21, 1932). A lo anterior se sumó el aumento de las basuras, los animales muertos en las calles, y los hedores barriales compuestos por innumerables tolderías al pie de los cerros.

Son incontables los decretos de la Gobernación que surgieron para detener el descontrol social y sanitario en masividad. La "vigilancia sanitaria de los sospechosos de enfermedades (...) y la desinfección de todas las habitaciones" (AGT, oficio № 23, 1932), marcaron la pauta política y policiaca para dichos objetivos disciplinantes y profilácticos en los barrios tocopillanos. La llegada de los pampinos generó que los conventillos en Tocopilla aumentaran su insalubridad y hacinamiento. La llamada Vivienda Barata -la solución habitacional-

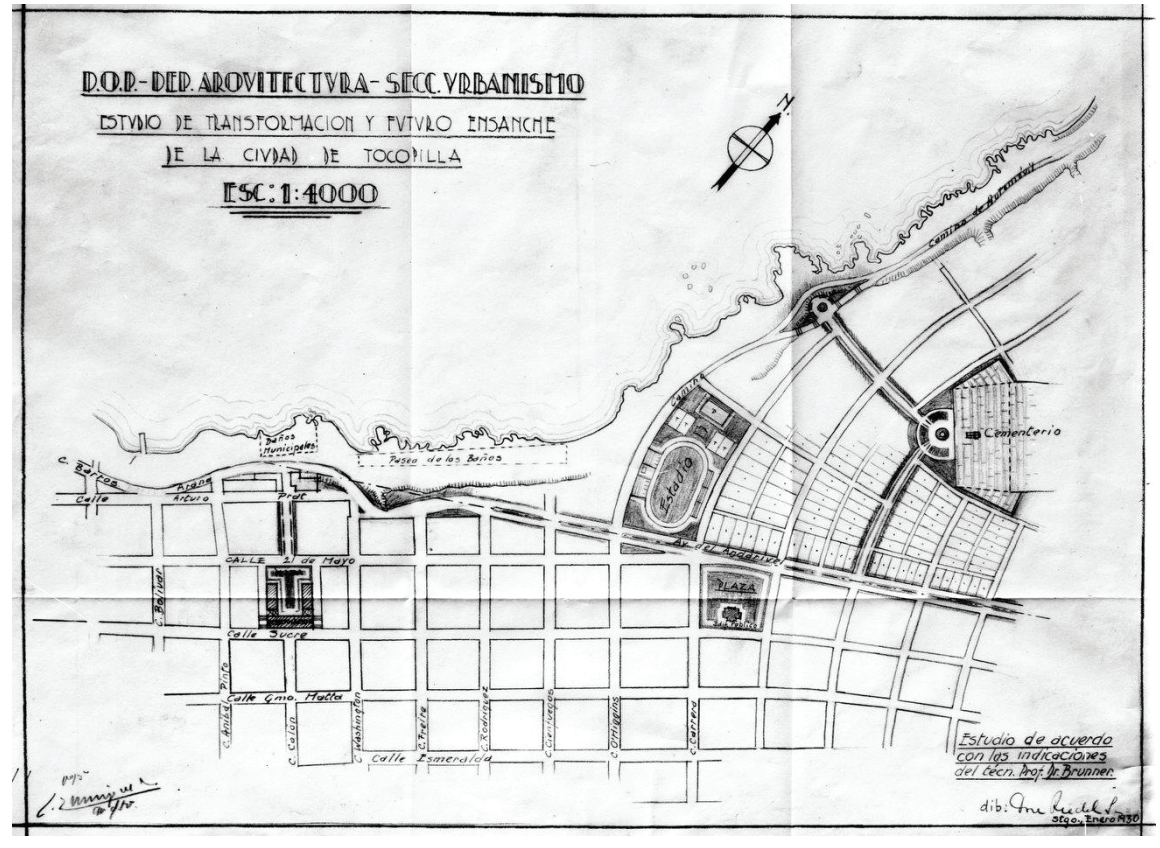

Imagen 1. Plano llamado Estudio de transformación y futuro ensanche de la ciudad de Tocopilla, elaborado de acuerdo con las indicaciones del Técnico Prof. Karl Brunner. Plano firmado por el arquitecto Luis Muñoz Maluschka. Los edificios Colectivos de la Dirección de Arquitectura del Ministerio de Obras Públicas de Chile).

tuvo su mayor impulso en el período comprendido entre 1930-1932, momento en que surgieron improvisados barrios de barracas, con casetas construidas toscamente y con materiales ligeros. En la mayoría de los casos, los obreros recibían del municipio la concesión de un terreno, derivándose la autoconstrucción de viviendas dentro de precarias posibilidades, donde se usaba como materiales sacos, cartones, madera, y se cubrían las frágiles paredes con diarios y papel de cajón de manzanas.

Todo este proceso de ocupación espacial y emergencia sanitaria desarmó las proyecciones del arquitecto y urbanista austriaco Karl Brunner Von Lehenstein², quien, junto a Luis Muñoz Maluschka ${ }^{3}$, visitó Tocopilla el 30 noviembre de 1929, para componer un plan de ensanche moderno y reubicar algunos equipamientos públicos ${ }^{4}$. El urbanista austriaco afirmó: "Tocopilla es por el momento una ciudad pequeña, pero tiene las bases para un desarrollo grande $y$, seguramente, tendrá un buen porvenir, pues las minas cercanas le permitirán llevar una próspera vida" (La Prensa de Tocopilla, 14 de diciembre 1929). Esta declaración, claramente, no vaticinaba el caos que generaría la gran depresión económica de EE.UU. y el cierre de las salitreras.

Superada parcialmente la crisis económica en el primer lustro de la década de 1930, hubo que administrar y controlar a ese gran segmento de la población que habitó espontáneamente los márgenes urbanos. La llegada del Frente Popular en 1938, inició un plan de gestión política y social, que articuló urbanismo y arquitectura moderna, entendiendo esta última como un dispositivo higienicista y disciplinario que diseñó una nueva impronta en el desierto costero. Dicho plan se orientó, sustancialmente, a reubicar e integrar al

${ }^{2}$ Ingeniero y arquitecto austríaco titulado en la Technische Hochschule de Viena, que realizó destacadas obras e intervenciones urbanísticas en varias ciudades de Chile y Colombia

${ }^{3}$ Arquitecto y urbanista chileno (1896-1974), quien realizó importantes aportes en la planificación urbana y ordenamiento territorial desde el Ministerio de Obras Públicas de Chile.

${ }^{4}$ Entre aquellos trabajos que se aprobaron en conjunto con la Gobernación, estuvo la implementación de una explanada cerca del muelle de la Compañía Salitrera Anglo Chilean; la ampliación

30 del Balneario Municipal; un paseo costero: el traslado del cementerio: la construcción de un parque anexo al futuro Estadio Municipal (construido en 1932); el ensanche de la Avenida Diagonal' y la

implementación de un campo de aviación. Brunner y Muñoz criticaron la ubicación del Matadero por estar cerca del Estadio (La Prensa de Tocopilla, 2 de diciembre de 1929).

${ }_{5}^{5}$ Alianza política conformada por el Partido Radical, el Comunista y el Socialista, adicionándose la Central de Trabajadores de Chile (CTCH), la Federación de Estudiantes de Chile (FECH) y el movimiento mapuche constituido en el Frente Único Araucano. 
sistema social a los obreros residentes en los barrios marginales. La idea era acabar con las barriadas y también se propuso educar y desinfectar a la población para ser parte del nuevo modelo de Estado y el proyecto político del Frente Popular. De ese modo, en el lapso que va desde 1939 hasta 1943, podemos registrar el surgimiento de una serie de inmuebles modernos que compusieron un conjunto urbano suburbial con una impronta social, política,

\section{LA IMPRONTA DE KULCZEWSKI.}

La nueva impronta del Frente Popular tuvo un claro referente: la figura de Luciano Kulczewski, arquitecto designado por el presidente Pedro Aguirre como administrador nacional de la Caja de Seguro Obrero Obligatorio el 13 de enero de 1939. En esas circunstancias, asistió al Primer Congreso de la Vivienda Popular en la ciudad de Buenos Aires en 1939, oportunidad en que realizó un crítico balance y diagnóstico sobre la situación que enfrentaba el problema de la vivienda en el país, cuestionando duramente las políticas y proyectos impulsados por la Caja de la Habitación Popular creada en 1936. Kulczewski señaló que "la causa principal ha sido el punto de partida erróneo de los planes: un criterio financiero y no económico social" (Kulczewski 1939: 7). En ese sentido, remarcó que las soluciones otorgadas por el Estado frente a las problemáticas habitacionales, solo reprodujeron la pobreza, la insalubridad y las patologías: "Se gasta ingenio y tinta en buscar soluciones que van desde el material inverosímil hasta planimetrías absurdas como nichos de cementerio; o se buscan terrenos que, por su precio, linden a páramos o basurales en los extremos de las ciudad creando a sus viviendas, nuevas y crueles diferencias" (Kulczewski 1939: 7).

Este criterio financiero en la solución habitacional, se alejaba completamente del propósito inicial, dando paso a trágicas fórmulas: "El conventillo-pueblo no puede romperse nunca mientras exista ésta política de (...) Conventillo-Tugurio, y como resultante Morbilidad y Mortalidad" (Kulczewski 1939: 6). Se trataba de binomios que venían a acrecentar solo resultados aterradores producto de la formación de barriadas, que evidenciaban el contraste entre "los felices y los infortunados, enrostrando una vez más a estos últimos su condición..." (Kulczewski 1939: 6).

Durante su período de gestión, Kulczewski instauró uno de sus proyectos más emblemáticos en la arquitectura nortina: la creación de conjuntos habitacionales colectivos para trabajadores, enmarcándose en la proyección de una vida salubre en aras de una mejor calidad de vida. Estos edificios colectivos vendrían a renovar la forma moderna de habitar de las ciudades del norte: Arica, Iquique, Antofagasta y Tocopilla. La modernidad tuvo su sello en la contextualización del diseño a la aridez del norte, zona que el arquitecto visitó en variadas oportunidades (Galaz-Mandakovic 2011). Dicho proyecto marcó el escenario para otros destacados arquitectos: Devilat, Aracena y Mönckeberg, significando una interesante impronta que dura hasta el

\section{tiempo presente.}

FRENTE POPULAR Y MODERNIDAD.

Para la ciudad de Tocopilla, la llegada del

Frente Popular significó la implementación de un interesante proyecto arquitectónico a través de un verdadero barrio moderno, que buscó nuevas formas de ocupar y morar en el desierto en el marco de una crisis social que debía ser resuelta desde el punto de vista habitacional, sanitaria y educacional. Todas estas arquitecturas modernas estaban situadas en el norte del borde urbano; es decir, en las proximidades de las barriadas populares surgidas por la crisis del salitre. Como arquitecturas modernas, el sello estuvo fundado en la racionalidad de sus diseños. Sus rasgos se encaminaron hacia las superficies lisas y sin ornamentos, también a los paños continuos, como crítica hacia los estilos decorativos que caracterizaron a la elite del Norte Grande, tales como el Art Decó y el Art Nouveau.

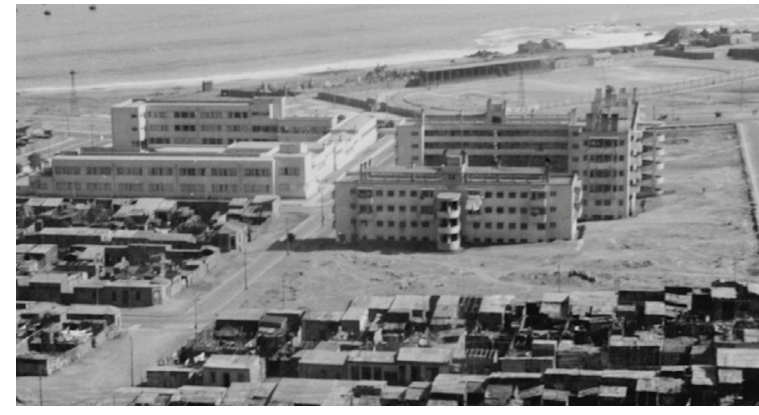

Imagen 2. Edificios Colectivos de la Caja del Seguro Obrero Obligatorio de Tocopilla, construidos entre 1939 y 1941 junto Establecimientos Educacionales (fuente: Archivo (c) Esteban Establecinipez).
Lón

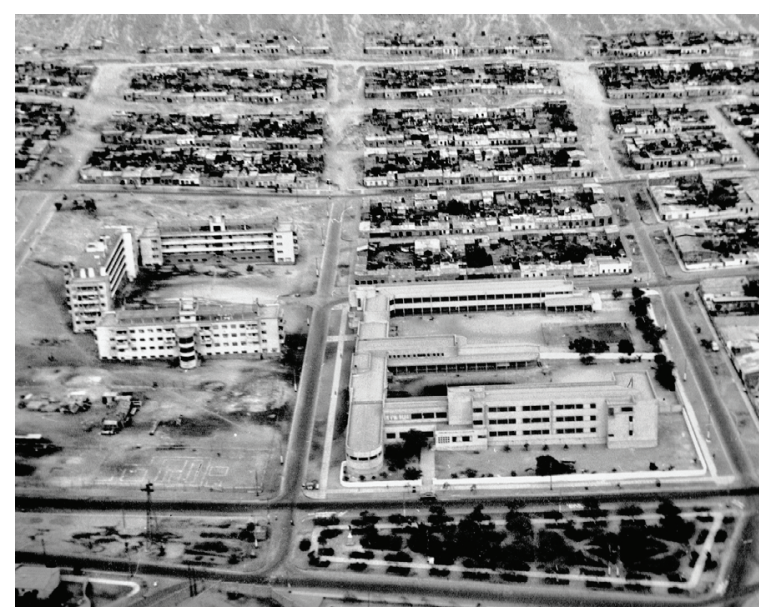

Imagen 3. Edificios Colectivos de la Caja del Seguro Obrero Obligatorio y el denominado Grupo Escolar, compuesto por la Escuela Superior de Hombres №7 y la Escuela Superior de Niñas Archivo La Prensa de Tocopilla). 
Una cuarta lectura nos sitúa en la constitución de una arquitectura moderna que representó a un Estado que destinó sus energías hacia el control social. Podemos comprender estos inmuebles en su condición de edificios máquinas, que se basaron en una esquematización desde el poder sobre el cómo debía ser la vida de los sectores populares. Para ello, la funcionalidad de estos inmuebles, particularmente los Edificios Colectivos de la Caja del Seguro Obrero Obligatorio, proyectados por Aquiles Zentilli bajo la dirección de Luciano Kulczewski, apostaron por el desarrollo de una vida en comunidad controlada. En ese sentido, la modernidad dialogó con la lógica del company town norteamericano, desarrollado en la minería del cobre y del salitre Guggenheim, proyectándose como estructuras disciplinantes.

Por tales razones, podemos leer este barrio como una reacción a un descontrol y a un desborde social generado por las poblaciones que llegaron desde la pampa salitrera del ciclo Shanks. Entonces, este tipo de arquitectura funcional y racional apostó por la constitución de un instrumento para gestionar y conducir la vida desde el Estado Una biopolítica ${ }^{8}$ que halló en el urbanismo y en la arquitectura sus dispositivos de acción, como parte de una estrategia política y general del poder (Foucault 2007a). Una estrategia centrada en el cuerpo fiscalizado como medio a un objetivo de disciplinamiento mayor

Los agentes del Estado fueron tras el control de una masa poblacional que, fruto de la cesantía, había dejado las estructuras de control social conocidas en la pampa salitrera. Una vez que llegan al puerto, fue necesario el control que abarcó desde la biología hasta la circulación y ubicación de los cuerpos en la localidad, en una clara cita al panóptico, sirviendo como laboratorio de técnicas para modificar la conducta o reeducar a los individuos, por lo que no solo fue un aparato de poder, sino también de saber. Surgieron, de ese modo, arquitecturas como máquinas pedagógicas, máquinas de acción médica y las máquinas de disciplinamiento colectivo y, en un costado, desde 1959, un reloj monumental, interpretado como dispositivo cronométrico del orden, disciplina y control, cardinal para la eficiencia en el trabajo, la integración social, económica y laboral, a lo que se agregó una iglesia católica y un parque ${ }^{9}$. Entonces, estas arquitecturas se proyectaron como efectivos operadores para la transformación de la población a través de la vigilancia y la constitución de un verdadero observatorio y encauzamiento. El lema de Pedro Aguirre Cerda, "Gobernar es Educar", fue la sinonimia de "Gobernar es encauzar". Encauzar dentro de un 'sistema de saberes', referido tanto a la conciencia de sí del propio gobierno como a los aparatos ideados por él para la producción de una sociabilidad que asegurara la inclusión de los ciudadanos en sus campos de administración. Se aplicó muy bien el concepto de gubernamentalidad ${ }^{10}$ (Foucault 2007b), porque se advirtieron las asimetrías en el acceso de los distintos actores al espacio político, mostrando el modo en que los más débiles terminaron entrando en la órbita hegemónica de los discursos dominantes a través de edificios que buscaron moldear la cotidianeidad. En esas circunstancias, es necesario fijar el análisis sobre la arquitectura moderna no solo en términos de materialidad y derechos sociales, sino que también en términos de tecnologías, tácticas y estrategias. Y es ahí donde la modernidad en el norte inauguró un devenir transformador desde una biopolítica.

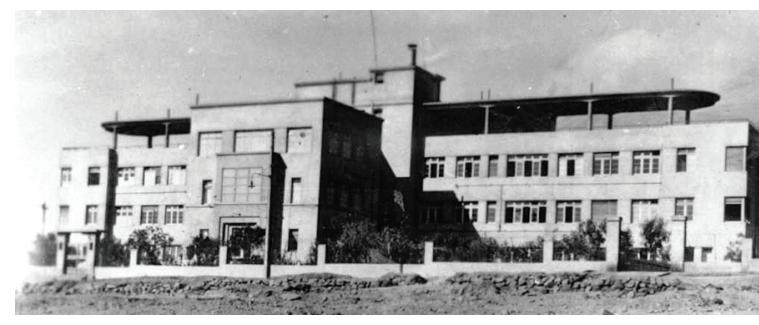

Imagen 5. Hospital Marcos Macuada Ogalde, edificio proyectado por el arquitecto Fernando Devilat Rocca (fuente: Archivo (c) La Prensa de Tocopilla).

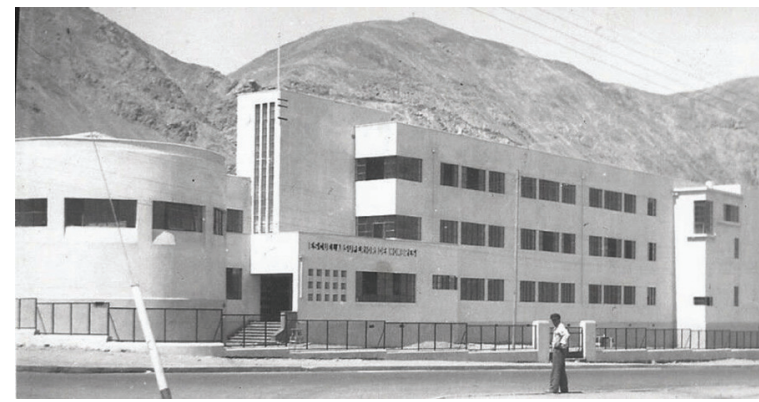

Imagen 6. Escuela Superior de Hombres № 1, edificio

Imantado por la Sociedad Constructora de Establecimientos Educacionales, que fue inaugurado en 1943 (fuente: Archivo of La Prensa de Tocopilla).

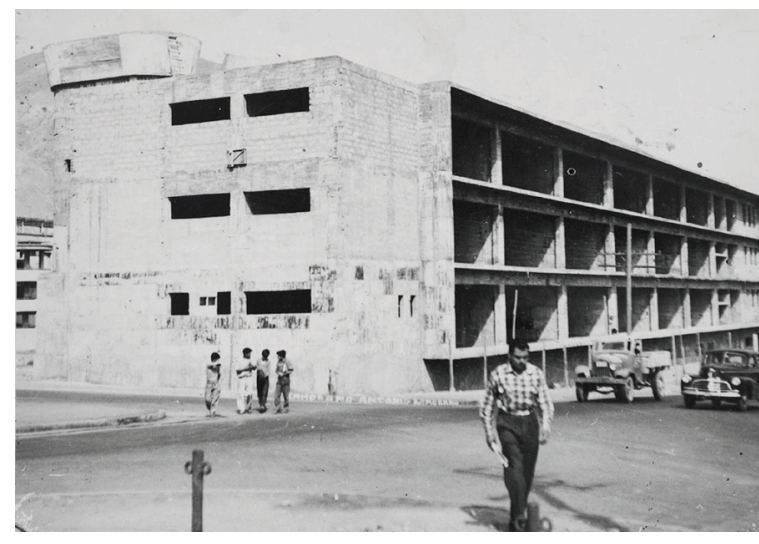

Imagen 7. Edificio tardomoderno del Liceo de Tocopilla proyectado por el arquitecto Carlos Albrecht. La firma construción se inicio en 1959 (fuente: Matte y CIA. Limitada. Su vo personal de Juan Chirino Michea, Tocopilla).

${ }^{8}$ Categoría que remite a la relación entre la política y la gestión de la vida de las poblaciones mediante una serie de dispositivos, tales como la higiene, la policía, la escuela, la medicina, las leyes y la estadistica para organizar los cuerpos e identificarlos. Entonces, la biopolítica deviene en una explosión de técnicas diversas y numerosas para obtener el sometimiento de los cuerpos y el control de las comunidades, sobre las cuales ya no basta la mera ideologia para controlarlas, sino que se adiciona lo biológico, lo somático y lo corporal (Foucault 2007 a).

${ }_{9}^{9}$ A propósito, Horacio Torrent, Moreno y Galaretto (2013), al hablar sobre la nuevas formas de la modernidad en arquitectura desde 1930, indican que aquellas estuvieron derechamente asociadas a un nuevo modelo de forma urbana: "la arquitectura como herramienta de transformación y de generación de un nuevo impulso de las ciudades. Dicho impulso estuvo en relación con el rol de la industria de la construcción en la reactivación económica posterior a la crisis de 1930. Más concretamente, con los esfuerzos que los sectores modernizantes de la sociedad llevaron adelante por establecer a la ciudad como maquinaria productiva" (Torrent et al. 2013: 10)

'La gubernamentalidad según Foucault (2007b), hace referencia a una economía específica de poder en las sociedades, donde el poder mismo es descentralizado haciendo que sus miembros jueguen un rol activo en su propio autogobierno. Debido a este rol activo, los individuos se regulan desde dentro. 
CONCLUSIÓN. Superado el proceso conocido como chilenización", surgido después de la Guerra del Pacífico (18791883), hubo que adicionar nuevos héroes para la república en el desierto de Atacama: el arquitecto, el profesor, el médico y el cura; todos ellos operaron dentro de una nueva batería de gestión estatal para construir un 'buen ciudadano', dignatario de la 'raza chilena'. Decidor es que el Frente Popular instauró el programa Defensa de la Raza y Aprovechamiento de las Horas Libres, mediante el cual se procuraba 'vigorizar y moralizar' a los ciudadanos mediante ejercicios y entretenimientos considerados honestos, con el aprovechamiento higiénico y educativo de las horas libres
(Aguirre 1942). Entonces, en este barrio se desfilaba, se aprendía, se rezaba, se jugaba (en el estadio), se vivía y se sanaban enfermedades. Se cruzó salud, educación, nacionalismo, deporte, catolicismo y control del tiempo. Era la expresión 'fiel' de un proyecto destinado a la construcción del 'buen vecino', útil para el país, el Estado y, por sobre todo, para el capitalismo minero que necesitaba reproducirse.

En el decir de Giorgio Agamben, la Zôe en Tocopilla se expresó como el simple hecho de un vivir silvestre, al borde de lo jurídico y de lo sanitario, a través de la espontaneidad de la ocupación espacial. Por ello, la urgencia de constituir un bíos, remitió a la necesidad de normar para así indicar "la forma o manera de vivir de individuo o grupo" (Agamben 2003: 9). Fue la urgencia para que dichas formas soberanas de vivir fuesen disipadas y esos cuerpos y sus acciones fueran ingresados en la polis, es decir, a la comunidad política. Lo anterior, con la finalidad de reproducir la posibilidad de un espacio para convivir en el marco de una arquitectura diseñada por el Estado, caracterizado por su paternalismo exuberante a través de la planificación, organización, integración, dirección y control de la población, la que tuvo que entrar en la órbita de las reglamentaciones estatales a través de la arquitectura del Movimiento Moderno, que prestó una clara utilidad, racionalidad y funcionalidad. $\mathbf{\Delta} \mathbf{s}$

\section{REFERENCIAS}

Agamben, G. 2003 Estado de excepción Homo Sacer Buenos Aires: Adriana Hidalgo Editora. Aguirre, J., 1942. Defensa de la Raza. 1939-1941. Santiago de Chile: Editorial Zig-Zag. Archivo de la Gobernación de Tocopilla - AGT, 1932. Of. № 23, 11 septiembre 1932 Archivo de la Gobernación de Tocopilla - AGT, 1932. Of. № 21, 22 septiembre 1932 Correa, S, Figueroa, C., Jocelyn-Holt, A., Rolle, C. y Vicuña, M., 2001. Historia del siglo XX chileno. Balance paradojal. Santiago de Chile: Editorial Sudamericana.

Foucault, M., 2007a. Nacimiento de la biopolitica. Buenos Aires: Fondo de Cultura Económica. Foucault, M., 2007b. Seguridad, territorio, población: Curso en el Collège de France. (1977-1978). Argentina: Fondo de Cultura Económica.

Galaz-Mandakovic, D., 2011. Edificios Colectivos de la Caja del Seguro Obrero Obligatorio, 1939-41. Movimiento moderno, solución social. Tocopilla: Retruécanos ediciones.
González S, 2002 Chilenizando a Tunupa La escuela pública en el Tarapacá Andino 1880-1990 Santiago de Chile: Ediciones de la Dirección de Bibliotecas, Archivos y Museos, Centro Barros Arana Kulczewski. L., 1939. "Discurso del Delegado Chileno D. Luciano Kulczewski, administrador de la Caja de Seguro Obrero Obligatorio, en el Primer Congreso de la Vivienda en Buenos Aires." Acción Social, 82.7. La Prensa de Tocopilla, 1932 (15 de mayo). Edición de mayo de 1932. La Prensa de Tocopilla, 1929 (14 de diciembre). Edición de diciembre de 1929 La Prensa de Tocopilla, 1929 (2 de diciembre). Edición de diciembre de 1929 La Prensa de Tocopilla, 1929 (noviembre). Edición de noviembre de 1929. League of Nations, 1933. World economic survey, 1932-33. Ginebra: League of Nations Torrent, H., Moreno, N. y Galaretto, L., 2013. “La arquitectura moderna en la producción de la gran ciudad: Chile 1930-1970." Anales de Investigación en Arquitectura, vol. 3. Universidad ORT Uruguay. 